\title{
Treatment Modality with Concurrent Chemo-Radiation for Locally Advanced Carcinoma Cervix, Four Field Technique EBRT Is Better Responsive than Two Field Technique
}

\author{
Md. Zillur Rahman Bhuiyan", ", Mamun Or Rasheed ${ }^{1}$, Sadia Sharmin', Moshiur Rahman², \\ Samia Ahmed ${ }^{2}$ \\ ${ }^{1}$ Department of Oncology, Bangabandhu Sheikh Mujib Medical University, Dhaka, Bangladesh \\ ${ }^{2}$ Department of Radiation Oncology, National Institute of Cancer Research Hospital, Dhaka, Bangladesh
}

Email address:

mdfaisal_81@ymail.com (Md. Z. R. Bhuiyan)

*Corresponding author

\section{To cite this article:}

Md. Zillur Rahman Bhuiyan, Mamun Or Rasheed, Sadia Sharmin, Moshiur Rahman, Samia Ahmed. Treatment Modality with Concurrent Chemo-Radiation for Locally Advanced Carcinoma Cervix, Four Field Technique EBRT Is Better Responsive than Two Field Technique. Cancer Research Journal. Vol. 8, No. 2, 2020, pp. 20-25. doi: 10.11648/j.crj.20200802.11

Received: January 4, 2020; Accepted: March 19, 2020; Published: April 23, 2020

\begin{abstract}
Background: Carcinoma of the cervix is the fourth most common cancer in women in the world.In 2018, an estimated 570000 women were diagnosed with cervical cancer worldwide and about 311000 women died from the disease. Bangladesh has the highest level of incidence and mortality rates due to cervical cancer among women due to lack of screening, high prevalence of risk factors and poor socio-economic condition. Methods: In this prospective observational study total 90 number of subjects were enrolled to compare the effectiveness of external beam radiation therapy by four field box technique versus two field technique in the treatment of locally advanced carcinoma of uterine cervix. Clinically and histologically diagnosed squamous cell carcinoma of uterine cervix patients were selected as study population. Results: In this study the highest number of patients were in between 46 to 55 years. Most of the subject used Oral contraceptive pill more than 5 years. Most of the patients were in stage IIB.Most of the patients were presented with postcoital bleeding, anemia, vaginal discharge , loss of appetite, Intermenstrual bleeding and pelvic pain. After completion of treatment follow-up per vaginal examination revealed that $73.33 \%$ subjects had complete response in four field group (Arm A). In two field group (Arm B), 57.78\% had complete response. Common toxicities related to treatment were nausea, vomiting, diarrhea, skin reaction, anemia, leucopenia, bladder toxicity and rectal toxicity. Conclusion: From this study it can be concluded that four field box technique in EBRT was statistically effective in relieving symptoms and achieving complete response than two field technique in the treatment of locally advanced carcinoma of uterine cervix.
\end{abstract}

Keywords: RT (Radiotherapy), EBRT (External Beam Radiation Therapy), CCRT (Concurrent Chemo-Radiation), ICRT (Intracavitary Radiotherapy/Brachytherapy)

\section{Introduction}

Carcinoma cervix is the eight most common cancer in the world. The incidence is very high in developing and underdeveloped countries. Women belonging to low socioeconomic status show higher incidence [13]. Carcinoma of uterine cervix is a malignant neoplasm of the cervix in which the cells of the cervix become abnormal and begin to grow uncontrollably, forming tumors. The cervix projects into the vaginal vault. Adjacent structures are the anterior, posterior, and lateral vaginal fornices. Anteriorly the cervix is related to the base of the bladder and posteriorly to the rectum and pouch of Douglas. Laterally it is related to the uterus [1]. The junction between the primary columnar epithelium, of the ectocervix and squamous epithelium of the ectocervix is the site, where cervical carcinoma develops [3]. Viral infection of the cervix may lead to premalignant change which is called dysplasia. Cervical dysplasia is graded mild, moderate to severe. The terms CIN-1, CIN-2, CIN-3, are histological term used to describe increasing degree of dysplasia. Most severe 
form of dysplasia, CIN-3 (previously called carcinoma in situ), will only progress to invasive cancer in between $20 \%$ and $40 \%$ of women if untreated. Over $90 \%$ of carcinoma of uterine cervix are squamous cell carcinoma. Approximately $7 \%$ to $9 \%$ are classified as adenocarcinoma, and $1 \%$ to $2 \%$ are clear cell and mesonephric type [2].

Cancer of the cervix is one of the most common cancer in women in the world, with an estimated 529,409 new cases and 274,883 deaths in 2008 [16]. It is the most common malignancy in South African black colored females with a lifetime risk of 1 in 41 and the 2nd and 5lh most common cancer in Asian and white females, respectively [14]. The incidence and mortality rates vary in different countries, the highest rates being recorded in the developing countries [7]. In Europe, variation between countries is higher in incidence than in mortality. In 2002, age- standardized incidence rates varied between the lowest rate 4.3 per 100,000 people per year in Finland and the highest of 27.4 per 100,000 people per year in Serbia Montenegro. Age-standardized mortality rates varied between 1.6 per 100.000 people per year in Malta and 13.0 per 100,000 people per year in Romania [7].

It is the most frequent cancer in women in developing countries, about $78 \%$ of cases occurring there, where cervical cancer is the most frequent cause of cancer death in women. Although the mean age of diagnosis is 52 years, carcinoma of the uterine cervix can be found in women between the ages of 17 to 90 years [12]

According to hospital based cancer registry carcinoma cervix is the second most common cancer among female in Bangladesh. Though there is no available national based cancer registry but some hospital based registry showed that every year about 25.000 women were diagnosed as carcinoma of uterine cervix. Mortality is very high, nearly half of them die annually. Frequency of carcinoma of uterine cervix is $22-29 \%$ [9]. Over $80 \%$ of the cervical cancer presents at a fairly advanced stage with five years survival rates less than $40 \%$. International federation of Gynecology and obstetrics (FIGO) clinical stage of disease at present is the most important predictor of longterm survival. Recurrences more than five years of treatment are extremely rare [5].

A number of factors may influence the choice of local treatment for cervical cancer, including tumor size, stage, histological feature, evidence of lymph node metastasis, risk factors for complications of surgery or radiotherapy patients performance status and patient preference. [3]

Patient with cervical carcinoma should be treated in close collaboration with the gynecologic oncologist and the radiation oncologist, and an integrated team approach should be vigorously pursued [6]. Invasive cervical cancer may be treated by surgery, radiotherapy or in combination of both, with or without chemotherapy [1].

Surgical treatment (radical hysterectomy) is effective management for early stage of cervical cancer. Cervical carcinoma is one of the malignancy that have been effectively treated with radiotherapy. Concurrent chemoradiation (CCRT) should be considered in preference to radiation alone in the treatment of early stage of carcinoma uterine cervix with poor prognostic factors after surgery and in the locally advanced cervical carcinoma. Chemotherapy is not used for primary disease as first line of treatment for cervical cancer [10].

Radiotherapy (RT) plays a major role in the treatment of invasive uterine cervical carcinoma. Controversy continues between those who advocate radical surgery and those who advocate radiation therapy for the treatment of early carcinoma of the uterine cervix, results are equivalent [2].

RT is the treatment of choice for FIGO stage IIB, III A, IIIB and IVA carcinoma of the cervix and is an excellent alternative to surgery in selected patients with stage IA, IB, or IIA diseases [4].

RT for primary cervical cancer consists of a combination of external beam radiotherapy (EBRT) and brachytherapy (BT), except in stage IA disease where BT alone may be used [4]. Ideally, pelvic radiotherapy begins with external beam radiation therapy, which is designed to shrink the primary tumor and to improve the geometry for the BT insertions that follow.

EBRT treats the whole pelvis, including clinically and radio logically apparent tumor, uterine corpus, upper part of vagina, parametrium, and the draining lymph nodes. The central disease (cervix, vagina, and parametrium) is further boosted by [17] Conventionally, EBRT planning is based on standard bony landmarks using X-rays and can be delivered by anterior-posterior and posterior- anterior (AP-PA) parallel opposed fields or the four field box technique. The conventional four field box technique with parallel opposed AP-PA fields and two lateral opposed fields achieves better dose distribution than the parallel opposed AP- PA field technique in terms of tumor coverage and a relatively reduced dose to normal tissue.

BT was first used to treat cervical cancers in the early $20^{\text {th }}$ century and continues to play a central role in their curative management. The goal of BT is to deliver a high dose to disease in the cervix and paracervical tissues while preserving function of adjacent critical structures [3].

The role of EBRT by four field box technique or by two field technique with associated acute toxicity for achieving complete response in the treatment of locally advanced carcinoma of uterine cervix were evaluated in this study.

\section{Methods}

This was a prospective observational study with two comparison group which include EBRT by four field box technique in Arm-A and two field technique in Arm-B with a view to observe and compare the effects, response rate and clinical outcome by two different technique of EBRT. All the patients of both arms were treated with external beam radiation, 50 Gy in 25 fractions over five weeks, concurrently cisplatin $30 \mathrm{mg} / \mathrm{m}^{2}$ i. v. weekly for 5 weeks. 45 patients (Arm A) received radiation by four field box technique and another 45 patients (Arm B) by two field technique during EBRT. A total 90 patients, who were histologically and radiologically diagnosed cases of locally advanced carcinoma cervix (stageIIB-IVA) were enrolled in this study. Permission from the concern department was taken for the study. Informed consent was taken from the patients before enrolling in the study All the 
patients in both arm received total 3 insertions of intracavitary brachytherapy after completion of EBRT. This study conducting in Bangabandhu Sheikh Mujib Medical University (BSMMU), Dhaka Medical College Hospital (DMCH) and National Institute of Cancer Research Hospital (NICRH).

\section{Results}

In this study the age of the patients were from 26-65 years. The highest number was found between 46 to 55 years of age group and the mean age was 47.5 years. Majority of the study subjects were from poor socio-economic class and most of the study subjects were illiterate. Most of the subject used Oral contraceptive pill more than 5 years. Smoker was present in both groups. Most of the patients were in stage IIB. Patients were presented with postcoital bleeding, anemia, vaginal discharge, loss of appetite. Intermenstrual bleeding and pelvic pain. After completion of treatment follow-up per vaginal examination revealed that $73.33 \%$ subjects had complete response in four field group (Arm A). In two field group (Arm B), 57.78\% had complete response. Common toxicities related to treatment were nausea, vomiting, diarrhea, skin reaction, anemia, leucopenia, bladder toxicity and rectal toxicity.

Table 1. Age distribution of the patients.

\begin{tabular}{lll}
\hline \multirow{2}{*}{ Age in years } & Arm A $(\mathbf{n}-\mathbf{4 5})$ & Arm B $(\mathbf{n}-\mathbf{4 5})$ \\
\cline { 2 - 3 } & No. of patients & No. of patients \\
\hline $26-35$ & $06(13.33 \%)$ & $04(08.89 \%)$ \\
$36-45$ & $08((17.78)$ & $05(11.11 \%)$ \\
$46-55$ & $18(40.00 \%)$ & $21(46.66 \%)$ \\
$56-65$ & $13(28.89 \%)$ & $15(33.33 \%)$ \\
Mean \pm SD & $46.28 \pm 18.58$ & $47.12 \pm 16.89$ \\
\hline
\end{tabular}

Table 2. Socio-economic status of the patients.

\begin{tabular}{lll}
\hline \multirow{2}{*}{ Socio-economic status } & Arm A (n-45) & Arm B (n-45) \\
\cline { 2 - 3 } & No. of patients & No. of patients \\
\hline Poor & $30(66.66 \%)$ & $27(60.00 \%)$ \\
Lower middle class & $09(20.00 \%)$ & $12(26.66 \%)$ \\
Middle class & $03(06.66 \%)$ & $05(11.11 \%)$ \\
Rich & $03(06.66 \%)$ & $01(03.33 \%)$ \\
\hline
\end{tabular}

Table 3. Risk factors of the patients.

\begin{tabular}{lll}
\hline \multirow{2}{*}{ Risk factors } & Arm A (n - 45) & Arm B (n-45) \\
\cline { 2 - 3 } & No. of patients & No. of patients \\
\hline OCP & $19(42.22 \%)$ & $21(46.66 \%)$ \\
Smoking & $11(24.44 \%$ & $06(13.33 \%)$ \\
Unhealthy personal hygiene & $15(33.33 \%)$ & $18(40.00 \%)$ \\
\hline
\end{tabular}

Table 4. Distribution of the patients with FIGO stage of disease at the time of diagnosis.

\begin{tabular}{lll}
\hline \multirow{2}{*}{ FIGO stage of disease } & Arm A (n-45) & Arm B (n-45) \\
\cline { 2 - 3 } & No. of s patients & No. of patients \\
\hline Stage IIB & $19(42.22 \%)$ & $20(44.44 \%$ \\
Stage IIA & $11(24.44 \%)$ & $12(26.67 \%)$ \\
Stage IIIB & $12(26.67 \%)$ & $12(26.67 \%)$ \\
Stage IVA & $01(02.22 \%)$ & $01(02.22 \%)$ \\
\hline
\end{tabular}

Table 5. Clinical presentation of the patients.

\begin{tabular}{lll}
\hline \multirow{2}{*}{ Clinical presentation } & Arm A (n -45) & Arm B (n-45) \\
\cline { 2 - 3 } & No. of patients & No. of patients \\
\hline Post coital bleeding & $36(80.00 \%)$ & $33(73.33 \%)$ \\
Intermenstruai bleeding & $22(48.89 \%)$ & $21(46.67 \%)$ \\
Vaginal discharge & $26(57.78 \%)$ & $26(57.78 \%)$ \\
Pelvic pain & $24(53.33 \%)$ & $22(48.89 \%)$ \\
Urinary symptoms & $21(46.67 \%)$ & $18(40.00 \%)$ \\
Rectal symptoms & $12(26.57 \%)$ & $09(20.00 \%)$ \\
Loss of appetite & $26(57.78 \%)$ & $24(53.33 \%)$ \\
Anemia & $33(73.33 \%)$ & $35(77.78 \%)$ \\
\hline
\end{tabular}

*Multiple responses were elicited. 
Table 6. Distribution of the patients according to treatment response.

\begin{tabular}{|c|c|c|c|}
\hline \multirow{2}{*}{ Response } & $\operatorname{Arm} A(n-45)$ & Arm B (n-45) & \multirow{2}{*}{ Statistical test } \\
\hline & No. of patients & No. of patients & \\
\hline Complete & $33(73.33 \%)$ & $26(57.78 \%)$ & \multirow{2}{*}{0.024} \\
\hline Partial & $12(26.67 \%)$ & $19(42.22 \%)$ & \\
\hline
\end{tabular}

Table 7. Distribution of the respondents on basis of staging and response.

\begin{tabular}{|c|c|c|c|c|c|c|}
\hline \multirow[b]{2}{*}{ Stage } & \multicolumn{3}{|c|}{$\operatorname{Arm} A(n=45)$} & \multicolumn{3}{|c|}{$\operatorname{Arm~B~(n=45)~}$} \\
\hline & $\begin{array}{l}\text { No. of } \\
\text { patients }\end{array}$ & $\begin{array}{l}\text { Complete response } \\
\text { n/\% }\end{array}$ & Partial response $\mathbf{n} / \%$ & $\begin{array}{l}\text { No. of } \\
\text { patients }\end{array}$ & $\begin{array}{l}\text { Complete response } \\
\text { n/\% }\end{array}$ & Partial response $n / \%$ \\
\hline IIB & 19 & $16(84.21)$ & 03 (15.79) & 19 & $12(63.16)$ & 07 (36.84) \\
\hline IIIA & 13 & $10(76.92)$ & $03(23.08)$ & 13 & $07(53.85)$ & $06(46.15)$ \\
\hline IIIB & 12 & $07(58.33)$ & 05 (41.67) & 12 & 05 (41.67) & $07(58.33)$ \\
\hline IVA & 01 & $00(00.00)$ & 01(100.00) & 01 & $00(00.00)$ & $01(100.00)$ \\
\hline
\end{tabular}

Table 8. Distribution of the patients on treatment related toxicities.

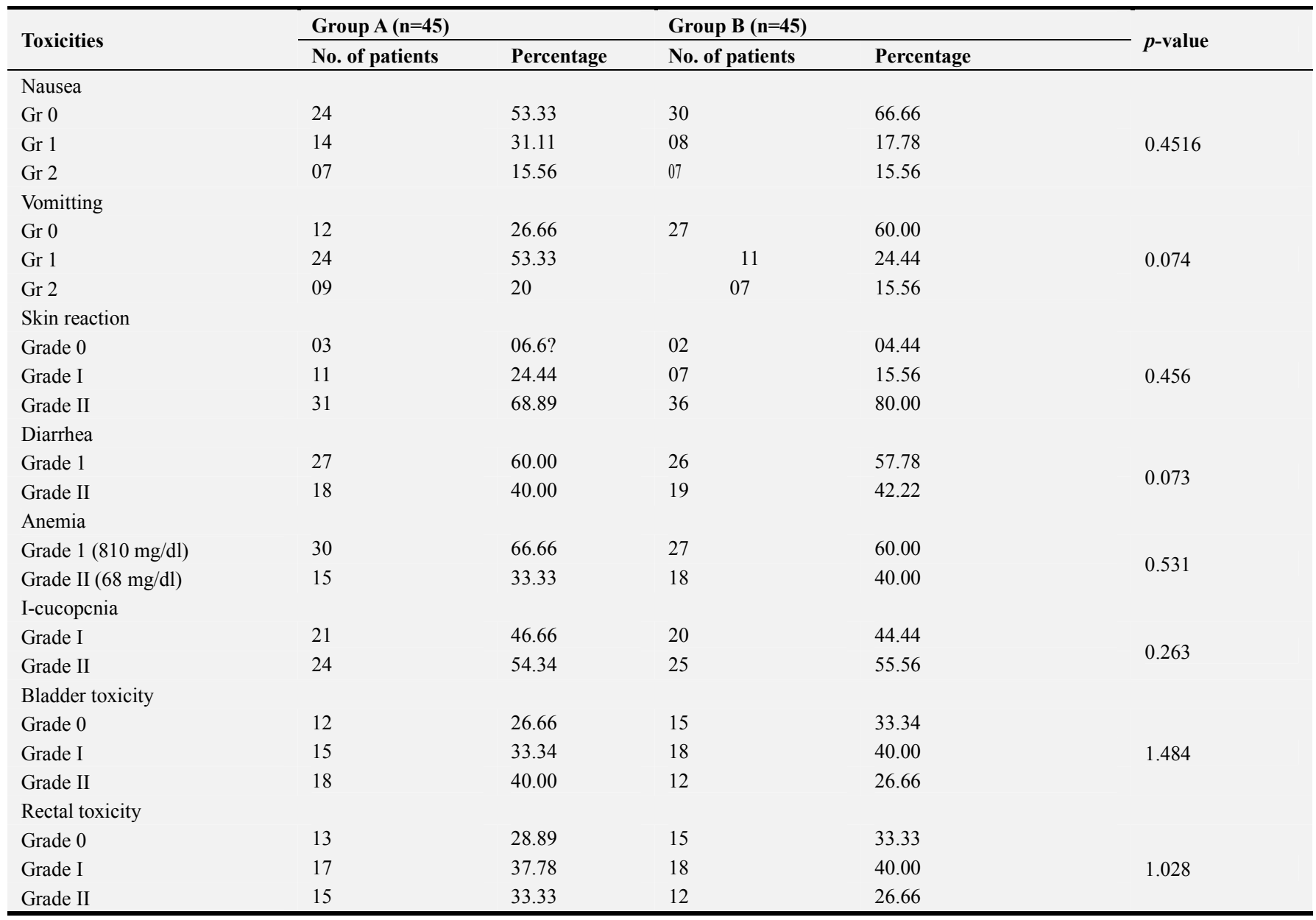

\section{Discussion}

Radiotherapy is an essential modality of treatment in Gynecology in the primary nonsurgical management and the adjuvant postoperative treatment of selected malignancies arising in the female reproductive tract. Current strategy for treating cancers of the uterine cervix depends on clinical and pathologic stage of disease. Early stage of the disease can be treated surgically if resection can be accomplished without substantial tissue disruption. RT is also a alternative choice of treatment in early stage of disease. Postoperative radiotherapy is reserved for the cases in which histopathologic analysis of the removed specimen reveals the features, suggesting a high risk local recurrence [8]. Primary RT can provide an opportunity for cure for women with unresectable, locally advanced disease; for women with resectable disease in whom the risk of surgical morbidity is unacceptably high; and for women with medical risk factors that contraindicate primary surgical therapy. However, for women with distant metastatic 
disease at presentation, cure is unlikely, although palliative RT frequently improves patient's quality of life when used to relieve symptoms. Although adjuvant radiotherapy is commonly used to manage advanced or metastatic cervical and endometrial cancer, it is sometimes administered as adjuvant therapy for ovarian cancer. Radiation therapy may also be used for hormonal ablation [11]. This current prospective observational study was carried out to compare the effectiveness of external beam radiation therapy by four field box technique versus two field technique in the treatment of locally advanced carcinoma of uterine cervix. In this prospective observational study from 90 subjects, 45 were in Arm A who were treated with four field box technique. In Arm $\mathrm{B}$ had also 45 subjects were treated with two field technique. The findings of the study are discussed on basis of related previous studies concerning the objective of the study. [18] Observed that early marriage, use of OCP, multiparity, low socio-economic condition, illiteracy, early age of intercourse were the common risk factors in elderly patient. Our present study revealed that majority of the study subjects were from poor socio economic class. Some of the study subjects in both arm were smoker \& unhealthy personal hygiene.

Because of direct extension of tumor into the adjacent structures limits the ability of surgery to gain clear surgical margins. BT is indicated for the management of stage IIB-IVA locally advanced cervical cancer [15]. All the present study subjects were between IIB-IVA. It was noted that most of the patients were in stage IIB (Arm-A 42.32\% vs. Arm-B 44.44\%).

Early stages of cervical cancer may be completely asymptomatic. Vaginal bleeding, post coital bleeding or a vaginal mass may indicate the presence of malignancy. Also, moderate pain during sexual intercourse and vaginal discharge are symptoms of cervical cancer. Symptoms of advanced cervical cancer may include loss of appetite, weight loss, fatigue, pelvic pain, back pain, leg pain, and swollen legs, heavy bleeding from the vagina, and/or leakage of urine or feces from the vagina. Similar study findings were observed in present study. In present study majority of the subjects with carcinoma of cervix presented with post coital bleeding (Arm A $80 \%$ vs. Arm B 73.33\%), anemia (Arm A 73.33\% vs. Arm B $77.78 \%$ ), vaginal discharge (57.78\%), loss of appetite (Arm A $57.78 \%$ vs. Arm B 53.33\%), intermenstrual bleeding (Arm A $48.89 \%$ vs. Arm B 46.67\%) and pelvic pain (Arm A 53.33\% vs. Arm B 48.89\%). per vaginal examination revealed $\mathrm{p} / \mathrm{v}$ bleeding, discharge and growth. These findings were also observed in per speculum findings. Per rectal examination revealed involvement of parametrium and fixation of pelvic floor.

EBRT treats the whole pelvis, including clinically and radiologically apparent tumor, uterine corpus, upper part of vagina, parametrium, and the draining lymph nodes. The central disease (cervix, vagina, and parametrium) is further boosted by intracavitary BT. Conventionally, EBRT planning is based on standard bony landmarks using X-rays and can be delivered by anterior-posterior and posterior- anterior (AP-PA) parallel opposed fields or the four field box technique.
The conventional four field box technique with parallel opposed AP-PA fields and two lateral opposed fields achieves better dose distribution than the parallel opposed AP-PA field technique in terms of tumor coverage and a relatively reduced dose to the normal tissues [11]. So, it was thought to be better than two field box technique. Similar findings were seen in present study. It was observed that $73.33 \%$ subjects had complete response in Arm A. In Arm B, 57.78\% had complete response. Statistical analysis revealed that there was significant difference in response between two arms.

Radiation to the pelvis can also irritate the bladder (radiation cystitis), causing discomfort and increases urinnary urge. Pelvic radiation can also lead to premature menopause. Skin changes arc also common which causes dry and moist desquamation.

Comparable toxicities related to treatment in present study were nausea, vomiting, skin reaction, diarrhea, anemia, leucopenia, bladder toxicity and rectal toxicity.

\section{Conclusion}

From this study it could be concluded that in the treatment modality of concurrent chemoradiation during four field box technique EBRT was statistically effective in relieving symptoms and achieving complete response than two field technique in the treatment of locally advanced carcinoma of uterine cervix. Acute toxicities were observed in this study and no statistical differences were found between the two treatment technique. So considering the relief of symptoms and complete response the four field box technique may be better treatment option than two field technique for the treatment of locally advanced carcinoma of uterine cervix.

\section{References}

[1] Bomford CK \& Kunkler III (eds) 2003, Walter and Miller's Textbook of Radiotherapy, 6th edition, Edinburgh London Churchil Livingstone.

[2] Chao KS, Perez CA, Brady LW \& Marinette T 2011, Radiation Oncology: management decisions, 3rd edition, Lippincott Williams \& Wilkins, Philadelphia.

[3] Devita VT Jr., Lawrence TS, Rosenberg SA. Cancer: Principles \& Practice of Oncology, 10th edition, 2015. Lippincott Williams \& Wilkins, New York.

[4] Duenas-G A, Cetina L, Mariscal ! and Garza J 2003, "Modem Management of Locally Advanced Cervical Carcinoma: Cancer Treatment Reviews", vol. 29. pp. 389-399.

[5] FIGO Committee on Gynecologic Oncology 2009. "Revised FIGO staging for carcinoma of the vulva, cervix, and endometrium". International Journal of Gynecology and Obstetrics, vol. 105, pp. 103-104.

[6] Halperin EC, Perez CA, Brady LW 2013, Perez and Brady's Principles and Practice of Oncology, 6th edition; Lippincot Williams \& Wilkins. 
[7] IARC 2007, International Agency for Research on Cancer and Globocan. Available at http://wwwdep.iarc.fr/.

[8] Keys MM, Roberts JA, Brunetto VL, 2004. A phase III trial of surgery with or without adjunctive external pelvic radiation therapy in intermediate risk endometrial adenocarcinoma: a Gynecologic Oncology Group study. Gynecol Oncol, vol 92. no. 3, pp. 744-51.

[9] Maula KJ 2010, "Recent managemen of cervical cancer. 22nd AGM and Scientific seminar-2010 (2011. February 5)` Ba lgladesh cancer society, pp. 36.

[10] Pazdur R, Wagman LD, Camhauscn KA, Hoskins WJ. Cancer Management: A Multidisciplinary Approach, 13th edition, 2010 UBM Medica LLC, New York.

[11] Randall ME, Filiaci VL, Muss H, Spirtos NM, Mannel RS, Fowler J 2006,. "Randomized phase III trial of whole-abdominal irradiation versus doxorubicin and cisplatin chemotherapy in advanced endometrial carcinoma": a Gynecologic Oncology Group Study. J Clin Oncol, vol. 24, no. 1, pp. 36-40.

[12] Rubin P, Williams JP. Cli cal Oncology, A Multidisciplinary Approach for Physicians and Students, 8th edition, 2001 W. B. Saunders Company, Newyork. London, Sydney.
[13] Segnan N, "Socioeconomic status and cancer screcning,"IARC Scientific Publication, no. 138, pp 369-376, 1997.

[14] Sitta F \& Madhoo J 1998, Cancer statistics in South Africa. Incidence of Histological Diagnoses of Cancer 1993-1995. National Cancer Registry of South Africa, Institute of Medical Research, JH.

[15] Stewart AJ, Viswanathan AN 2006. Current controversies in high-dose-ratc versus low-dose-rate brachytherapy for cervical cancer vol. 107, no. 5, pp. 908-15.

[16] WHO/ICO Innformation center on HPV and Cervical Cancer (HPV information Center). Human papillomavirus and related cancers in Bangladesh. Summary report 2010.

[17] Xunino S, Rosato O, Lucino S, Jaur gui E, Rossi L, Venencia D. Anatomic study of the pelvis in carcinoma of uterine cervix as releated to the box technique. Int J Radial Oncol Biol Phys 1999, vol. 44, pp. 53-59.

[18] Zhang X, Dai, B. Zhang. B. Wang, Z 2011, Risk of cervical cancer: A meta-analysis. Gynecologic oncology vol. 124. no. 2, pp. 366-73. 